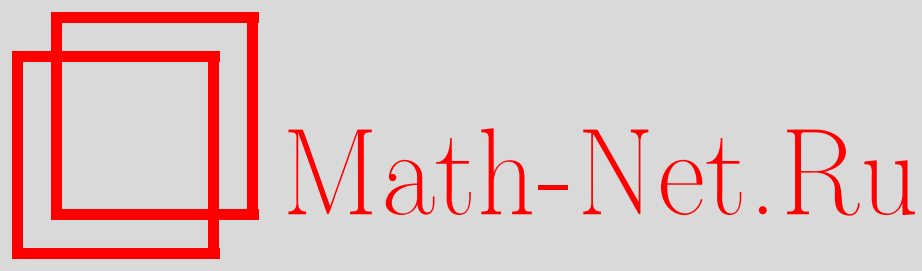

М. Арджери, В. Бароне, С. Де Лилло, Д. Лупо, М. Соммакал, Существование минимумов энергии для упругих тонких стержней в статических спиральных конфигурациях, ТМФ, 2009, том 159, номер 3, 336-352

DOI: https://doi.org/10.4213/tmf6354

Использование Общероссийского математического портала Math-Net.Ru подразумевает, что вы прочитали и согласны с пользовательским соглашением http://www.mathnet.ru/rus/agreement

Параметры загрузки:

IP : 54.209 .52 .79

26 апреля 2023 г., 15:21:04

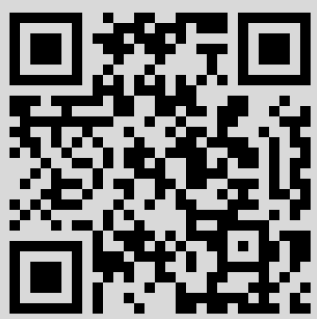




\title{
ФИЗИКА
}

Том 159, № 3

июнь, 2009

\section{СУЩЕСТВОВАНИЕ МИНИМУМОВ ЭНЕРГИИ ДЛЯ УПРУГИХ ТОНКИХ СТЕРЖНЕЙ В СТАТИЧЕСКИХ СПИРАЛЬНЫХ КОНФИГУРАЦИЯХ}

\begin{abstract}
Дана физическая характеризация семейств решений статической модели Кирхгофа тонкого упругого стержня, порождающих широкий спектр возможных спиральных конфигураций и зависящих от поведения так называемого регистра, а также от значений радиуса и шага спирали, существование которых было доказано ранее. Описана плотность энергии для каждого из этих решений в терминах свойств упругости и геометрической формы стержня без напряжений, что позволяет определить механизм отбора для выявления предпочтительных спиральных конфигураций. Этот анализ может стать фундаментальным средством в понимании тесной связи между исследованием упругих деформаций в тонких стержнях и крупномасштабными моделями, имеющими широкое применение в естественных науках.
\end{abstract}

Ключевые слова: тонкий упругий стержень, уравнение Кирхгофа, обратная задача, интегрируемость, спираль.

\section{1. ВВЕДЕНИЕ}

В последнее время колоссальные достижения квантово-механических методов и алгоритмов нашли свое отражение в возрастающем количестве численных исследований, посвященных структурному и конформационному поведению биополимеров, начиная с фундаментальных повторяющихся элементов и переходя к низшим олигомерам и бесконечным полимерам [1]. В результате этих исследований были получены не только полезные данные для параметризации и подтверждения классических силовых полей (так называемые атомистические подходы, широко используемые в численной молекулярной динамике), но и фундаментальные указания на

${ }^{*}$ Dipartimento di Chimica and INSTM-Village, Università Federico II, Complesso Univ. MSA, Via Cintia, I-80126 Napoli, Italy.

${ }^{\dagger}$ Scuola Normale Superiore Pisa, Pisa; INFN, Sezione di Perugia, Perugia, Italy.

E-mail: vincenzo.barone@sns.it

${ }^{\ddagger}$ Dipartimento di Matematica e Informatica and INSTM-Village, Università degli Studi di Perugia, Via Vanvitelli 1, 06123 Perugia, Italy, Istituto Nazionale di Fisica Nucleare, Sezione di Perugia, Perugia, Italy. E-mail: silvana.Delillo@pg.infn.it 
химические эффекты, ответственные за устойчивость наиболее важных вторичных структур, таких как $\alpha$ - и 310 -спирали, менее компактизованные структуры, а также различные виды спиральных витков для случая белков [2], [3]. В настоящее время широко признано, что равновесная структура биополимеров возникает в результате тонкого баланса между взаимодействиями различных классов, обусловленными взаимодействиями между соседними и удаленными остатками и эффектами среды [4]. Хотя наиболее распространенными вторичными структурами биополимеров являются спирали, они не всегда отвечают минимумам энергии для повторяющихся элементов, и некоторые численные исследования действительно наводили на мысль, что дальнодействующие кооперативные диполь-дипольные взаимодействия являются критическими для стабилизации спиральных структур. Структура биополимеров в целом подвергается дальнейшему изменению за счет других конкретных эффектов, таких как стэкинговые взаимодействия между ароматическими составляющими, электростатическое и стерическое взаимодействие между боковыми цепями, ионные взаимодействия и т.д. Однако фундаментальные причины предрасположенности к образованию растянутых или спиральных структур можно, видимо, понять с помощью моделей с низким разрешением, включающих характеристические и микроскопические процессы, которые нельзя распространить на макроскопические объекты и/или медленные явления. Это приводит к развитию крупномасштабных и мезоскопических моделей, параметры которых выводятся из микроскопических численных экспериментов [5]. Однако все эти численные подходы требуют новых сложных вычислений при изменении некоторых характеристик исследуемой системы, а нахождение фундаментальных закономерностей поведения основано на интуиции и терпеливых проверках. Альтернативный способ действий, которому мы следуем в настоящей работе, состоит в переходе непосредственно к аналитически решаемым моделям, в которых и локальные, и дальнодействующие взаимодействия моделируются простыми функциями, зависящими от длины дуги, которые, в свою очередь, выводятся из результатов микроскопических численных экспериментов. В работе [6] мы показали, что модель упругого стержня можно применить для случая анизотропных модулей упругости, что приводит к интегрируемым уравнениям, допускающим цилиндрические спиральные решения. В настоящей работе мы покажем, что в этой модели могут возникнуть протяженные или спиральные минимумы энергии, зависящие от отношения между продольным и поперечным модулями упругости. Таким образом, можно индуцировать переходы между различными структурами в целом путем модифицикации свойств упругости конкретных остатков за счет, например, изменения свойств среды. Например, как показывают детальные молекулярно-динамические вычисления [7], изменения рН могут приводить к протонированию некоторых остатков, а это, в свою очередь, может изменить их анизотропию упругости, что далее может привести к модифицикации спиральных характеристик. Настоящая работа посвящена статическим аспектам этой задачи, а именно описанию энергетического ландшафта, получающегося из различных модулей упругости. Это дает нам необходимые исходные данные для начала динамических исследований, направленных на изучение переходов между различными 


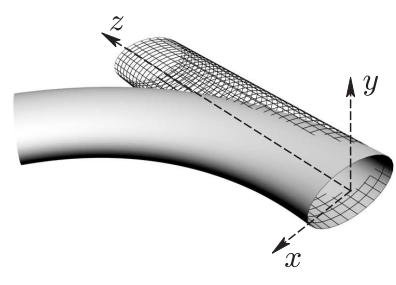

$(x, z)$-изгиб

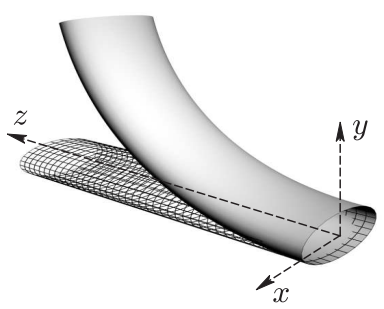

$(y, z)$-изгиб

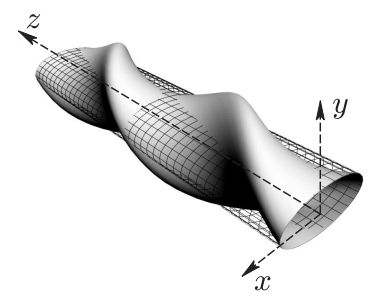

кручение

Рис. 1. Разрешенные деформации упругого стержня с эллиптическим поперечным сечением.

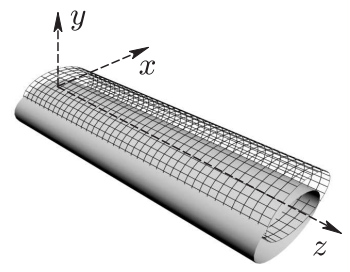

сдвиг

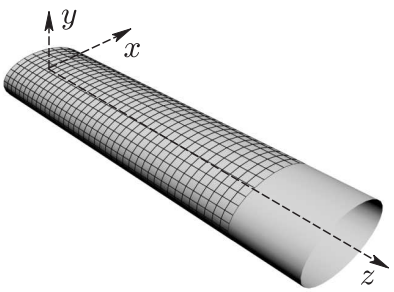

растяжение

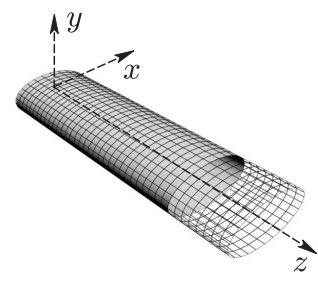

сжатие

Рис. 2. Запрещенные деформации упругого стержня с эллиптическим поперечным сечением.

структурами, что, как можно надеяться, приведет к лучшему пониманию фундаментальных процессов в биологических системах, таких как сворачивание и разворачивание белков.

\section{2. УРАВНЕНИЯ КИРХГОФА ДЛЯ УПРУГИХ СТЕРЖНЕЙ}

Напомним основные свойства статической модели Кирхгофа для упругого стержня (см. [8]-[10]), при этом мы будем руководствоваться работой [6].

Упругий стержень представляет собой деформируемый трехмерный объект, длина которого в продольном направлении предполагается большей, чем длина вдоль поперечного направления; при этом продольная ширина может быть конечной или бесконечной, но поперечная ширина всегда конечна. Как и в работе [6], в нашей модели рассматриваются деформации упругого стержня, полученные в результате изгиба и кручения (рис. 1), и запрещены деформации, полученные в результате продольного сдвига, расширения или сжатия (рис. 2).

Важно заметить, что при отсутствии деформации растяжения-сдвига форма поперечного сечения стержня может меняться в зависимости от длины дуги $s$.

Рассмотрим гладкую кривую в обычном евклидовом пространстве $\mathbb{R}^{3}$,

$$
\vec{r}(s): I \subseteq \mathbb{R} \rightarrow \mathbb{R}^{3},
$$

проходящую через центроиды поперечных сечений стержня. 
Чтобы задать положение поперечного сечения в общем положении, введем обобщенный ортонормальный репер Френе $\left\{\vec{d}_{1}(s), \overrightarrow{d_{2}}(s), \overrightarrow{d_{3}}(s)\right\}$, где $\vec{d}_{1}(s)$ и $\vec{d}_{2}(s)$ определяют плоскость поперечного сечения, а $\vec{d}_{3}(s)$ касателен к кривой $\vec{r}(s)$, предполагая отсутствие деформации растяжения-сдвига. Вариация вектора $\vec{d}_{i}(s), i=1,2,3$, в зависимости от длины дуги выражается в терминах вектора Дарбу

$$
\vec{k}(s)=\sum_{i=1}^{3} k_{i}(s) \vec{d}_{i}(s),
$$

где $k_{1}(s)$ и $k_{2}(s)$ связаны с изгибом, а $k_{3}(s)-$ с кручением, так что

$$
\frac{d \vec{d}_{i}(s)}{d s}=\vec{k}(s) \times \vec{d}_{i}(s), \quad i=1,2,3 .
$$

При каждом значении $s \in I$ можно определить базис Френе $\{\vec{N}(s), \vec{B}(s), \vec{T}(s)\}$, образованный нормальным, бинормальным и касательным векторами к кривой $\vec{r}(s)$. Обобщенный и обычный реперы Френе связаны вращением вокруг вектора $\overrightarrow{d_{3}}(s)$ на угол $\varphi(s)$ :

$$
\begin{aligned}
& \vec{d}_{1}(s)=\cos \varphi(s) \vec{N}(s)+\sin \varphi(s) \vec{B}(s), \\
& \vec{d}_{2}(s)=-\sin \varphi(s) \vec{N}(s)+\cos \varphi(s) \vec{B}(s), \\
& \vec{d}_{3}(s)=\vec{T}(s) .
\end{aligned}
$$

Следуя терминологии, принятой в исследованиях ДНК [11], назовем угол $\varphi(s)$ регистром. Напомним, что при отсутствии деформации растяжения-сдвига геометрическая конфигурация упругого стержня будет полностью описана, если задать элементы вектора Дарбу $\vec{k}$ в виде

$$
k_{1}(s)=\kappa(s) \sin \varphi(s), \quad k_{2}(s)=\kappa(s) \cos \varphi(s), \quad k_{3}(s)=\tau(s)+\frac{d \varphi(s)}{d s}
$$

где

$$
\kappa(s)=\left|\frac{d \vec{T}(s)}{d s}\right|, \quad \tau(s)=-\frac{1}{\kappa(s)} \frac{d \vec{T}(s)}{d s} \cdot \frac{d \vec{B}(s)}{d s}
$$

- соответственно кривизна и кручение вектора $\vec{r}(s)$, которые являются функциями от $s$, а $d \varphi(s) / d s$ - внутренний твист, являющийся мерой различия между плотностью твиста и кручением Френе.

В равновесии при отсутствии внешних импульсов и сил получаем статические уравнения Кирхгофа

$$
\begin{gathered}
\frac{d \vec{F}(s)}{d s}=0 \\
\frac{d \vec{M}(s)}{d s}+\vec{d}_{3}(s) \times \vec{F}(s)=0,
\end{gathered}
$$


где

$$
\vec{F}(s)=\sum_{i=1}^{3} F_{i}(s) \vec{d}_{i}(s)
$$

- результирующие упругие напряжения,

$$
\vec{M}(s)=\sum_{i=1}^{3} M_{i}(s) \vec{d}_{i}(s)
$$

- результирующий вращательный момент, действующий на поперечное сечение B $\vec{r}(s)$.

Вращательный момент $\vec{M}(s)$ связан с переменными напряжения линейным соотношением

$$
\vec{M}(s)=a_{1}(s) k_{1}(s) \vec{d}_{1}(s)+a_{2}(s) k_{2}(s) \vec{d}_{2}(s)+b(s) k_{3}(s) \vec{d}_{3}(s)
$$

где функции $a_{1}(s)$ и $a_{2}(s)$ - изгибные жесткости, являющиеся мерой асимметрии поперечного сечения, $b(s)$ - торсионная жесткость, являющаяся реакцией стержня на напряжение твиста.

Проецируя уравнения (7) на репер $\left\{\vec{d}_{i}(s)\right\}$ и используя (8), получаем систему Кирхгофа для шести неизвестных $\left(k_{1}, k_{2}, k_{3}\right)$ и $\left(F_{1}, F_{2}, F_{3}\right)$, рассматриваемых как функции длины дуги $s$ :

$$
\begin{gathered}
F_{1}^{\prime}+k_{2} F_{3}-k_{3} F_{2}=0, \\
F_{2}^{\prime}+k_{3} F_{1}-k_{1} F_{3}=0, \\
F_{3}^{\prime}+k_{1} F_{2}-k_{2} F_{1}=0, \\
F_{2}+\left(a_{2}-b\right) k_{2} k_{3}-a_{1} k_{1}^{\prime}-k_{1} a_{1}^{\prime}=0, \\
F_{1}+\left(a_{1}-b\right) k_{1} k_{3}+a_{2} k_{2}^{\prime}+k_{2} a_{2}^{\prime}=0, \\
k_{3}^{\prime} b+k_{3} b^{\prime}-\left(a_{1}-a_{2}\right) k_{1} k_{2}=0 .
\end{gathered}
$$

В большинстве практических задач по моделированию биологических и синтетических полимеров основной интерес представляют круговые спирали (т.е. $\alpha$-спирали, $3_{10}$-спирали, $\beta$-тяжи и т.д., см. [3]), соответствующие круговым спиральным решениям уравнений (9) (см. [6]). В настоящей работе мы рассмотрим решения такого типа, для которых характерны постоянные кривизна и кручение, а потому и постоянный шаг спирали.

\section{3. ПЛОТНОСТЬ ЭНЕРГИИ}

Плотность энергии тонких упругих стержней, описываемых системой (9) (см., например, [8]-[14]), имеет вид

$$
\mathcal{E}=\frac{1}{2}\left(a_{1} k_{1}^{2}+a_{2} k_{2}^{2}+b k_{3}^{2}\right)+F_{3} .
$$

Первое слагаемое в выражении (10) - это вклад изгибных и торсионных деформаций, а последнее слагаемое возникает из-за присутствия объемных внешних сил 
вдоль стержня. Важно заметить, что $\mathcal{E}$ имеет физическую размерность энергии на единицу длины, а именно размерность силы. Более того, дифференцируя (10) по $s$ и используя (9), отмечаем, что $\mathcal{E}$ не зависит от длины дуги $s$, если

$$
a_{1}^{\prime} k_{1}^{2}+a_{2}^{\prime} k_{2}^{2}+b^{\prime} k_{3}^{2}=0
$$

Предположим, что центральная линия рассматриваемого стержня является круговой спиралью, параметризованной векторной функцией $\vec{P}(t): \mathbb{R} \rightarrow \mathbb{R}^{3}$ :

$$
\vec{P}(t)=\{r \cos t, r \sin t, c t\}, \quad t \in\left[-\frac{\ell}{\sqrt{c^{2}+r^{2}}}, \frac{\ell}{\sqrt{c^{2}+r^{2}}}\right],
$$

где

$$
r=\frac{\kappa}{\kappa^{2}+\tau^{2}}, \quad c=\frac{\tau}{\kappa^{2}+\tau^{2}},
$$

$r$ - радиус спирали, $p=2 \pi c-$ шаг, $2 \ell$ - длина стержня (которая может также быть бесконечной). Для такой конфигурации, если $\kappa \neq 0$, энергия деформации для $N$ витков равна

$$
E=\int_{0}^{2 \pi N \sqrt{r^{2}+c^{2}}} \mathcal{E} d s
$$

где учтена трансляционная инвариантность уравнений (9) по отношению к длине дуги $s$. Если $\mathcal{E}$ не зависит от длины дуги, то легко вычислить упругую энергию на один виток спирали, вычисляя интеграл (14) на одном витке, полагая $N=1$.

Из элементарных геометрических рассуждений следует, что если $\kappa=0$, то параметризация (12) уже не применима и спираль вырождается в прямолинейный отрезок длины $2 \ell$, кручение которого (выражающее некомпланарность кривой в пространстве $\mathbb{R}^{3}$ ) можно положить равным нулю. В этом случае нельзя определить энергию на один виток, поэтому приходится изменить верхний и нижний пределы в интеграле (14):

$$
E=\int_{-\ell}^{\ell} \mathcal{E} d s
$$

\section{4. АНАЛИТИЧЕСКИЕ РЕШЕНИЯ}

В работе [6] авторы формулируют и решают следующую обратную задачу: если регистр $\varphi(s)$ фиксирован и для $\kappa(s)$ и $\tau(s)$ выбраны постоянные значения

$$
\kappa(s) \equiv \kappa, \quad \tau(s) \equiv \tau,
$$

то система (9) решается как система для шести неизвестных $a_{1}(s), a_{2}(s), b(s), F_{1}(s)$, $F_{2}(s), F_{3}(s)$ при фиксированных значениях конкретной длины дуги $s_{0}=0$ (уравнения (9) автономны и инвариантны относительно трансляций по $s$ ). Все возможные решения этой обратной задачи можно разбить на восемь семейств, зависящих от постоянных значений $\kappa(s)$ и $\tau(s)$ и от поведения регистра $\varphi(s)$.

1. При $\tau \neq 0$ и $\kappa \neq 0$ необходимо рассмотреть следующие три случая:

a) $\varphi(s) \equiv n \pi / 2, n \in \mathbb{Z}$; 
б) $\varphi(s)=\varphi_{0}-\tau s$;

в) $\varphi(s)$ не относится ни к одному из предыдущих случаев.

2. При $\tau=0$ и $\kappa \neq 0$ необходимо рассмотреть следующие три случая:

а) $\varphi(s) \equiv n \pi / 2, n \in \mathbb{Z}$;

б) $\varphi(s) \equiv \varphi_{0}$ при $\varphi_{0} \neq n \pi / 2, n \in \mathbb{Z}$;

в) $\varphi(s)$ не является постоянной функцией.

3. При $\tau=0$ и $\kappa=0$ необходимо рассмотреть следующие два случая:

а) $\varphi(s) \equiv \varphi_{0}$

б) $\varphi(s)$ не является постоянной функцией.

В настоящей работе мы рассматриваем все указанные выше случаи без доказательства (более подробно см. работу [6]). Кроме того, в каждом из случаев мы кратко опишем плотность энергии в терминах радиуса, шага и физических параметров (жесткостей и регистра) спирали.

4.1. СлучАй 1а: $\tau \neq 0, \kappa \neq 0$ и $\varphi(s) \equiv n \pi / 2, n \in \mathbb{Z}$. В этом случае можно найти решение в явном виде. Целесообразно рассмотреть по отдельности случаи четного и нечетного $n \in \mathbb{Z}$.

4.1.1. Пусть $n=2 m, m \in \mathbb{Z}$. Тогда

$$
\begin{array}{llrl}
a_{1}(s) \in \mathcal{C}^{1}(\mathbb{R}), & a_{2}(s)=(-1)^{m+1} \frac{F_{2}(0)}{\kappa \tau}+b_{0}, & b(s) & =b_{0}, \\
k_{1}(s)=0, & k_{2}(s)=(-1)^{m} \kappa, & k_{3}(s)=\tau, \\
F_{1}(s)=0, & F_{2}(s)=F_{2}(0), & F_{3}(s)=(-1)^{m} \frac{\tau F_{2}(0)}{\kappa} .
\end{array}
$$

Напомним, что $a_{1}(s)>0 \forall s$ при фиксированном $a_{1}(0), b_{0}=b(0)$ - вещественная положительная константа; $b_{0}$ и $F_{2}(0)$ должны быть выбраны так, чтобы $a_{2}(s)>0 \forall s$.

С учетом (10) и (13) можно явно записать плотность энергии

$$
\mathcal{E}=\frac{1}{2}\left((-1)^{m} F_{2}(0) \frac{2 c^{2}-r^{2}}{r c}+\frac{b_{0}}{r^{2}+c^{2}}\right) .
$$

Заметим, что плотность энергии (18) не зависит от длины дуги и от изгибной жесткости $a_{1}(s)$. Более того, подчеркнем, что изгибная жесткость $a_{2}(s)$ в $(17)$ явно зависит от кривизны $\kappa$ и от кручения $\tau$, откуда следует, что если варьировать радиус и шаг, то будет изменяться не только плотность энергии (18), но и внутренняя конфигурация стержня. Тогда для изучения поведения энергии в зависимости от $r$ и $c$ для конкретной конфигурации стержня необходимо задать $F_{2}(0)$ таким образом, чтобы $a_{2}(s)$ не зависело от кривизны и кручения.

4.1.2. Пусть $n=2 m+1, m \in \mathbb{Z}$. Тогда

$$
\begin{aligned}
& a_{1}(s)=(-1)^{m} \frac{F_{1}(0)}{\kappa \tau}+b_{0}, \quad a_{2}(s) \in \mathcal{C}^{1}(\mathbb{R}), \quad b(s)=b_{0}, \\
& k_{1}(s)=(-1)^{m+1} \kappa, \quad k_{2}(s)=0, \quad k_{3}(s)=\tau, \\
& F_{1}(s)=F_{1}(0), \quad F_{2}(s)=0, \quad F_{3}(s)=(-1)^{m+1} \frac{\tau F_{1}(0)}{\kappa} \text {. }
\end{aligned}
$$


Напомним, что $a_{2}(s)>0 \forall s$ при фиксированном $a_{2}(0), b_{0}=b(0)$ - вещественная положительнаая постоянная; $b_{0}$ и $F_{1}(0)$ должны быть выбраны так, чтобы $a_{1}(s)>0 \forall s$.

С учетом (10) и (13) можно явно записать плотность энергии для этого случая:

$$
\mathcal{E}=\frac{1}{2}\left((-1)^{m} F_{1}(0) \frac{2 c^{2}-r^{2}}{r c}+\frac{b_{0}}{r^{2}+c^{2}}\right) .
$$

Заметим, что плотность энергии (20) не зависит от длины дуги и от изгибной жесткости $a_{2}(s)$. Аналогично п. 4.1.1 изгибная жесткость $a_{1}(s)$ в (19) явно зависит от кривизны $\kappa$ и от кручения $\tau$, откуда следует, что если варьировать радиус и шаг, то будет изменяться не только плотность энергии (20), но и внутренняя конфигурация стержня. Тогда для изучения поведения энергии в зависимости от $r$ и $c$ для конкретной конфигурации стержня необходимо задать $F_{1}(0)$ таким образом, чтобы $a_{1}(s)$ не зависело от кривизны и кручения.

4.2. СлУЧАЙ 1б: $\tau \neq 0, \kappa \neq 0$ и $\varphi(s)=\varphi_{0}-\tau s$.

4.2.1. Пусть $\kappa \neq \tau$. Тогда

$$
\begin{gathered}
a_{1}(s)=\frac{\alpha \kappa^{2}}{\kappa^{2}-\tau^{2}}, \quad a_{2}(s)=\frac{\alpha \kappa^{2}}{\kappa^{2}-\tau^{2}}, \quad b(s) \in \mathcal{C}^{1}(\mathbb{R}), \\
k_{1}(s)=\kappa \sin \left(\varphi_{0}-\tau s\right), \quad k_{2}(s)=\kappa \cos \left(\varphi_{0}-\tau s\right), \quad k_{3}(s)=0, \\
F_{1}(s)=-\frac{\alpha \kappa^{3} \tau}{\kappa^{2}-\tau^{2}} \sin \left(\varphi_{0}-\tau s\right), \\
F_{2}(s)=-\frac{\alpha \kappa^{3} \tau}{\kappa^{2}-\tau^{2}} \cos \left(\varphi_{0}-\tau s\right), \quad F_{3}(s)=-\frac{\alpha \kappa^{2} \tau^{2}}{\kappa^{2}-\tau^{2}},
\end{gathered}
$$

где $\alpha$ - вещественная постоянная такая, что $a_{1}(s)$ и $a_{2}(s)$ - положительные вещественные постоянные, $b(s)>0 \forall s$ при фиксированном $b(0)$.

С учетом (10) и (13) можно явно записать плотность энергии для этого случая:

$$
\mathcal{E}=\frac{\alpha r^{2}\left(r^{2}-2 c^{2}\right)}{2\left(r^{2}-c^{2}\right)\left(r^{2}+c^{2}\right)^{2}} .
$$

Заметим, что плотность энергии (22) не зависит от длины дуги и от торсионной жесткости $b(s)$. Более того, подчеркнем, что изгибные жесткости $a_{1}(s)$ и $a_{2}(s)$ в $(21)$ явно зависят от кривизны $\kappa$ и от кручения $\tau$, откуда следует, что если варьировать радиус и шаг, то будет изменяться не только плотность энергии (22), но и внутренная конфигурация стержня. Тогда для изучения поведения энергии в зависимости от $r$ и $c$ для конкретной конфигурации стержня необходимо задать $\alpha$ таким образом, чтобы $a_{1}(s)$ и $a_{2}(s)$ не зависели от кривизны и кручения.

4.2.2. Пусть $\kappa=\tau$. Тогда

$$
\begin{gathered}
a_{1}(s)=-\frac{F_{3}(0)}{\kappa^{2}}, \quad a_{2}(s)=-\frac{F_{3}(0)}{\kappa^{2}}, \quad b(s) \in \mathcal{C}^{1}(\mathbb{R}), \\
k_{1}(s)=\kappa \sin \left(\varphi_{0}-\kappa s\right), \quad k_{2}(s)=\kappa \cos \left(\varphi_{0}-\kappa s\right), \quad k_{3}(s)=0, \\
F_{1}(s)=F_{3}(0) \sin \left(\varphi_{0}-\kappa s\right), \quad F_{2}(s)=F_{3}(0) \cos \left(\varphi_{0}-\kappa s\right), \quad F_{3}(s)=F_{3}(0),
\end{gathered}
$$


где $F_{3}(0)$ - вещественная постоянная такая, что $a_{1}(s)$ и $a_{2}(s)$ - положительные вещественные постоянные, $b(s)>0 \forall s$ при фиксированном $b(0)$.

С учетом (10) и (13) можно явно записать плотность энергии для этого случая:

$$
\mathcal{E}=\frac{F_{3}(0)}{2}
$$

Заметим, что плотность энергии (24) не зависит от длины дуги и от изгибной жесткости $b(s)$. Тогда можно показать, что даже если регистр линеен по длине дуги (а именно, изгибное и торсионное напряжения не обращаются в нуль), единственный вклад в энергию упругости дают объемные внешние силы. Как и в п. 4.2.1 изгибные жесткости $a_{1}(s)$ и $a_{2}(s)$ в $(23)$ явно зависят от кривизны $\kappa$ и кручения $\tau$, откуда следует, что если варьировать радиус и шаг, то будет изменяться не только плотность энергии (24), но и внутренняя конфигурация стержня. Тогда для изучения поведения энергии в зависимости от $r$ и $c$ для конкретной конфигурации стержня необходимо задать $F_{3}(0)$ таким образом, чтобы $a_{1}(s)$ и $a_{2}(s)$ не зависели от кривизны и кручения.

4.3. СлУчАЙ 1в: $\tau \neq 0, \kappa \neq 0$ и $\varphi(s)$ не относятся ни к одному из предыдущих случаев. В этом случае

$$
\begin{gathered}
a_{1}(s)=\frac{\beta_{0}}{\tau}+\frac{2 \beta_{2}}{\kappa^{2}}\left(\cos s \omega \operatorname{ctg} \varphi(s)-\frac{1}{\sqrt{1+\gamma^{2}}} \sin s \omega\right), \\
a_{2}(s)=\frac{\beta_{0}}{\tau}-\frac{2 \beta_{2}}{\kappa^{2}}\left(\cos s \omega \operatorname{tg} \varphi(s)+\frac{1}{\sqrt{1+\gamma^{2}}} \sin s \omega\right), \\
b(s)=\frac{\beta_{1} \omega+2 \beta_{2} \sin s \omega}{\omega\left(\tau+\varphi^{\prime}(s)\right)}, \\
k_{1}(s)=\kappa \sin \varphi(s), \quad k_{2}(s)=\kappa \cos \varphi(s), \quad k_{3}(s)=\tau+\varphi^{\prime}(s), \\
F_{1}(s)=\kappa\left(\beta_{1}-\beta_{0}\right) \sin \varphi(s), \quad F_{2}(s)=\kappa\left(\beta_{1}-\beta_{0}\right) \cos \varphi(s), \quad F_{3}(s)=\tau\left(\beta_{1}-\beta_{0}\right),
\end{gathered}
$$

где

$$
\gamma=\frac{\kappa}{\tau}, \quad \omega=\sqrt{\kappa^{2}+\tau^{2}},
$$

$\beta_{0}, \beta_{1}, \beta_{2}$ - произвольные вещественные постоянные, связанные с начальными условиями для $a_{1}(s), a_{2}(s), b(s)$ и $\varphi(s)$ следующим образом:

$$
\begin{gathered}
\beta_{0}=\frac{\tau}{2}\left\{a_{1}(0)+a_{2}(0)-\left(a_{1}(0)-a_{2}(0)\right) \cos 2 \varphi(0)\right\}, \\
\beta_{1}=\left(\tau+\varphi^{\prime}(0)\right) b(0), \quad \beta_{2}=\frac{\kappa^{2}}{4}\left(a_{1}(0)-a_{2}(0)\right) \sin 2 \varphi(0) .
\end{gathered}
$$

Физическая характеризация величин $a_{1}(s), a_{2}(s), b(s)$ рассматривается в работе [6]. С учетом (10) и (13) можно явно записать плотность энергии для этого случая:

$$
\mathcal{E}=\frac{1}{2}\left[\frac{\beta_{0} r^{2}+\left(3 \beta_{1}-2 \beta_{0}\right) c^{2}}{c\left(r^{2}+c^{2}\right)}+\left(\beta_{1}+\frac{2 \beta_{2} \sin \frac{s}{\sqrt{r^{2}+c^{2}}}}{\sqrt{r^{2}+c^{2}}}\right) \varphi^{\prime}(s)\right] .
$$


Из (28) видно, что плотность энергии зависит от длины дуги и от структурных параметров $\beta_{0}, \beta_{1}$ и $\beta_{2}$. Более того, энергия, вычисленная по формуле (14) с помощью (28), зависит от поведения регистра, за исключением того случая, когда внутренний твист обращается в нуль (мы подробно рассмотрим этот частный случай в разделе 5$)$. В случае $\varphi^{\prime}(s)=0$ заметим, что постоянная $\beta_{2}$ не играет никакой роли в функциональном поведении энергии. Снова подчеркнем, что жесткости $a_{1}(s)$, $a_{2}(s)$ и $b(s)$ в $(25)$ явно зависят от кривизны $\kappa$ и от кручения $\tau$, откуда следует, что если варьировать радиус и шаг, то будет изменяться не только плотность энергии (28), но и внутренняя конфигурация стержня. В этом случае при выборе постоянных интегрирования и регистра в общем положении нет способа задать $\beta_{0}, \beta_{1}$ и $\beta_{2}$ таким образом, чтобы $a_{1}(s), a_{2}(s)$ и $b(s)$ не зависели от кривизны и кручения.

4.4. СЛУЧАЙ $2 \mathrm{a}: \tau=0, \kappa \neq 0$ и $\varphi(s) \equiv n \pi / 2, n \in \mathbb{Z}$.

4.4.1. Пусть $n=2 m, m \in \mathbb{Z}$. Тогда

$$
\begin{gathered}
a_{1}(s) \in \mathcal{C}^{1}(\mathbb{R}), \quad a_{2}(s)=a_{0}-\frac{(-1)^{m} F_{1}(0) \sin s \kappa+F_{3}(0) \cos s \kappa}{\kappa^{2}}, \quad b(s) \in \mathcal{C}^{1}(\mathbb{R}), \\
k_{1}(s)=0, \quad k_{2}(s)=(-1)^{m} \kappa, \quad k_{3}(s)=0, \\
F_{1}(s)=(-1)^{m+1} F_{3}(0) \sin s \kappa+F_{1}(0) \cos s \kappa, \quad F_{2}(s)=0, \\
F_{3}(s)=(-1)^{m} F_{1}(0) \sin s \kappa+F_{3}(0) \cos s \kappa,
\end{gathered}
$$

где $a_{1}(s)>0$ и $b(s)>0 \forall s$ при фиксированных $a_{2}(0)$ и $b(0), a_{0}=a_{2}(0)+F_{3}(0) / \kappa^{2}-$ произвольная вещественная постоянная такая, что $a_{0}>\sqrt{F_{1}(0)^{2}+F_{3}(0)^{2}} / \kappa^{2}$, где $F_{1}(0), F_{3}(0)$ - вещественные постоянные.

С помощью $(10),(13)$ и учитывая, что из $\tau=0$ следует $c=0$, можно явно записать плотность энергии для рассматриваемого случая:

$$
\mathcal{E}=\frac{1}{2}\left(\frac{a_{0}}{r^{2}}+F_{3}(0) \cos \frac{s}{r}+(-1)^{m} F_{1}(0) \sin \frac{s}{r}\right) .
$$

Из (30) видно, что плотность энергии явно зависит от длины дуги и не зависит от поведения изгибной и торсионной жесткостей $a_{1}(s)$ и $b(s)$. Более того, подчеркнем, что изгибная жесткость $a_{2}(s)$ из $(29)$ явно зависит от кривизны $\kappa$, откуда следует, что если варьировать радиус, то будет изменяться не только плотность энергии (30), но и внутренняя конфигурация стержня. В этом случае, при выборе постоянных интегрирования в общем положении, нет способа задать $F_{1}(0)$ и $F_{3}(0)$ таким образом, чтобы жесткость $a_{2}(s)$ не зависела от кривизны.

4.4.2. Пусть $n=2 m+1, m \in \mathbb{Z}$. Тогда

$$
\begin{gathered}
a_{1}(s)=a_{0}-\frac{(-1)^{m+1} F_{2}(0) \sin s \kappa+F_{3}(0) \cos s \kappa}{\kappa^{2}}, \quad a_{2}(s) \in \mathcal{C}^{1}(\mathbb{R}), \quad b(s) \in \mathcal{C}^{1}(\mathbb{R}), \\
k_{1}(s)=(-1)^{m} \kappa, \quad k_{2}(s)=0, \quad k_{3}(s)=0, \\
F_{1}(s)=0, \quad F_{2}(s)=(-1)^{m} F_{3}(0) \sin s \kappa+F_{2}(0) \cos s \kappa, \\
F_{3}(s)=(-1)^{m+1} F_{2}(0) \sin s \kappa+F_{3}(0) \cos s \kappa,
\end{gathered}
$$


где $a_{2}(s)>0$ и $b(s)>0$ для любого $s$ при фиксированных $a_{2}(0)$ и $b(0), a_{0}=$ $a_{1}(0)+F_{3}(0) / \kappa^{2}-$ произвольная вещественная постоянная такая, что $a_{0}>$ $\sqrt{F_{2}(0)^{2}+F_{3}(0)^{2}} / \kappa^{2}, F_{2}(0), F_{3}(0)$ - вещественные постоянные.

С помощью (10), (13), учитывая, что из $\tau=0$ следует $c=0$, можно явно записать плотность энергии для этого случая:

$$
\mathcal{E}=\frac{1}{2}\left(\frac{a_{0}}{r^{2}}+F_{3}(0) \cos \frac{s}{r}+(-1)^{m+1} F_{2}(0) \sin \frac{s}{r}\right) .
$$

Из (32) видно, что плотность энергии явно зависит от длины дуги и не зависит от поведения изгибной и торсионной жесткостей $a_{2}(s)$ и $b(s)$. Аналогично п. 4.4.1 изгибная жесткость $a_{1}(s)$ в (31) явно зависит от кривизны $\kappa$, откуда следует, что если варьировать радиус, то будет изменяться не только плотность энергии (32), но и внутренняя конфигурация стержня. В этом случае при выборе постоянных интегрирования в общем положении нет способа задать $F_{2}(0)$ и $F_{3}(0)$ таким образом, чтобы жесткость $a_{1}(s)$ не зависела от кривизны.

4.5. СлУчАй 2б: $\tau=0, \kappa \neq 0$ и $\varphi(s) \equiv \varphi_{0}$ при $\varphi_{0} \neq n \pi / 2, n \in \mathbb{Z}$. В этом случае

$$
\begin{gathered}
a_{1}(s)=\alpha_{0}+\alpha_{1} \cos s \kappa+\alpha_{2} \sin s \kappa, \\
a_{2}(s)=\alpha_{0}+\alpha_{1} \cos s \kappa+\alpha_{2} \sin s \kappa, \quad b(s) \in \mathcal{C}^{1}(\mathbb{R}), \\
k_{1}(s)=\kappa \sin \varphi_{0}, \quad k_{2}(s)=\kappa \cos \varphi_{0}, \quad k_{3}(s)=0, \\
F_{1}(s)=\kappa^{2}\left(\alpha_{1} \sin s \kappa-\alpha_{2} \cos s \kappa\right) \cos \varphi_{0}, \\
F_{2}(s)=-\kappa^{2}\left(\alpha_{1} \sin s \kappa-\alpha_{2} \cos s \kappa\right) \sin \varphi_{0}, \quad F_{3}(s)=-\kappa^{2}\left(\alpha_{1} \cos s \kappa+\alpha_{2} \sin s \kappa\right),
\end{gathered}
$$

где $a_{1}(s)>0, a_{2}(s)>0$ и $b(s)>0$ для любого $s$ при фиксированных $a_{1}(0), a_{2}(0)$ и $b(0), \alpha_{0}, \alpha_{1}, \alpha_{2}$ - такие вещественные постоянные, что $\alpha_{0}>\sqrt{\alpha_{1}^{2}+\alpha_{2}^{2}}$. Более того, $\alpha_{0}, \alpha_{1}, \alpha_{2}$ выражаются через начальные условия $a_{1}(0), a_{2}(0)$ и вектор $\vec{F}(0)$ следующим образом:

$$
\begin{gathered}
\alpha_{0}=a_{1}(0)+\frac{F_{3}(0)}{\kappa^{2}}=a_{2}(0)+\frac{F_{3}(0)}{\kappa^{2}}, \quad \alpha_{1}=-\frac{F_{3}(0)}{\kappa^{2}}, \\
\alpha_{2}=\frac{F_{2}(0)}{\kappa^{2} \sin \varphi_{0}}=-\frac{F_{1}(0)}{\kappa^{2} \cos \varphi_{0}} .
\end{gathered}
$$

С помощью (10), (13), учитывая, что из $\tau=0$ следует $c=0$, можно явно записать плотность энергии для этого случая:

$$
\mathcal{E}=\frac{1}{2 r^{2}}\left(\alpha_{0}-\alpha_{1} \cos \frac{s}{r}-\alpha_{2} \sin \frac{s}{r}\right) .
$$

Из (35) видно, что плотность энергии явно зависит от длины дуги и не зависит от поведения торсионной жесткости $b(s)$. Снова подчеркнем, что изгибные жесткости $a_{1}(s)$ и $a_{2}(s)$ в $(33)$ явно зависят от кривизны $\kappa$ и кручения $\tau$, откуда следует, что если варьировать радиус и шаг, то будет изменяться не только плотность энергии (35), но и внутренняя конфигурация стержня. В этом случае при выборе постоянных интегрирования в общем положении нет способа задать $\alpha_{0}, \alpha_{1}$ и $\alpha_{2}$ таким образом, чтобы $a_{1}(s)$ и $a_{2}(s)$ не зависели от кривизны и кручения. 
4.6. СлУЧАЙ 2в: $\tau=0, \kappa \neq 0$ и $\varphi(s)$ является не постоянной функцией. В этом случае

$$
\begin{gathered}
a_{1}(s)=\gamma_{0}+\gamma_{1} \cos s \kappa+\gamma_{2} \sin s \kappa+\left(\beta_{0} \cos s \kappa+\beta_{1} \sin s \kappa\right) \operatorname{ctg} \varphi(s), \\
a_{2}(s)=\gamma_{0}+\gamma_{1} \cos s \kappa+\gamma_{2} \sin s \kappa-\left(\beta_{0} \cos s \kappa+\beta_{1} \sin s \kappa\right) \operatorname{tg} \varphi(s), \\
b(s)=\frac{\kappa\left(\beta_{0} \sin s \kappa-\beta_{1} \cos s \kappa+\beta_{2}\right)}{\varphi^{\prime}(s)} \\
k_{1}(s)=\kappa \sin \varphi(s), \quad k_{2}(s)=\kappa \cos \varphi(s), \quad k_{3}(s)=\varphi^{\prime}(s), \\
F_{1}(s)=\beta_{2} \kappa^{2} \sin \varphi(s)+\kappa^{2}\left(\gamma_{1} \sin s \kappa-\gamma_{2} \cos s \kappa\right) \cos \varphi(s), \\
F_{2}(s)=\beta_{2} \kappa^{2} \cos \varphi(s)-\kappa^{2}\left(\gamma_{1} \sin s \kappa-\gamma_{2} \cos s \kappa\right) \sin \varphi(s) \\
F_{3}(s)=-\kappa^{2}\left(\gamma_{1} \cos s \kappa+\gamma_{2} \sin s \kappa\right)
\end{gathered}
$$

где $\gamma_{0}, \gamma_{1}, \gamma_{2}, \beta_{0}, \beta_{1}, \beta_{2}$ - вещественные постоянные, выражающиеся в терминах начальных условий для функций $a_{1}(s), a_{2}(s), b(s), \varphi(s)$ и вектора $\vec{F}(s)$ следующим образом:

$$
\begin{gathered}
\gamma_{0}=a_{2}(0)+\frac{F_{3}(0)}{\kappa^{2}}+\left(a_{1}(0)-a_{2}(0)\right) \sin ^{2} \varphi(0), \\
\gamma_{1}=-\frac{F_{3}(0)}{\kappa^{2}}, \quad \gamma_{2}=-\frac{1}{\kappa^{2}}\left(F_{1}(0) \cos \varphi(0)-F_{2}(0) \sin \varphi(0)\right), \\
\beta_{0}=\frac{1}{2}\left(a_{1}(0)-a_{2}(0)\right) \sin 2 \varphi(0), \\
\beta_{1}=\frac{1}{\kappa^{2}}\left(F_{1}(0) \sin \varphi(0)+F_{2}(0) \cos \varphi(0)\right)-\frac{b(0)}{\kappa} \varphi^{\prime}(0), \\
\beta_{2}=\frac{1}{\kappa^{2}}\left(F_{1}(0) \sin \varphi(0)+F_{2}(0) \cos \varphi(0)\right) .
\end{gathered}
$$

Физическая характеризация величин $a_{1}(s), a_{2}(s)$ и $b(s)$ рассмотрена в работе [6].

С помощью (10), (13), учитывая, что из $\tau=0$ следует $c=0$, можно явно записать плотность энергии для этого случая:

$$
\mathcal{E}=\frac{1}{2 r^{2}}\left(\gamma_{0}-\gamma_{1} \cos \frac{s}{r}-\gamma_{2} \sin \frac{s}{r}\right)+\frac{\varphi^{\prime}(s)}{2 r}\left(\beta_{2}-\beta_{1} \cos \frac{s}{r}+\beta_{0} \sin \frac{s}{r}\right) .
$$

Видно, что плотность энергии зависит от длины дуги и от двух наборов структурных параметров $\beta_{0}, \beta_{1}, \beta_{2}$ и $\gamma_{0}, \gamma_{1}, \gamma_{2}$. Более того, энергия (14) зависит от поведения регистра, за исключением того случая, когда внутренний твист обращается в нуль (мы подробно рассмотрим этот частный случай в разделе 5). Снова подчеркнем, что жесткости $a_{1}(s), a_{2}(s)$ и $b(s)$ в (36) явно зависят от кривизны $\kappa$ и кручения $\tau$, откуда следует, что если варьировать радиус и шаг, то будет изменяться не только плотность энергии (39), но и внутренняя конфигурация стержня. В этом случае при выборе постоянных интегрирования и регистра в общем положении нет способа задать $\gamma_{0}, \gamma_{1}, \gamma_{2}$ и $\beta_{0}, \beta_{1}, \beta_{2}$ таким образом, чтобы $a_{1}(s), a_{2}(s)$ и $b(s)$ не зависели от кривизны и кручения. 
4.7. СлУчАй $3 \mathrm{a}: \kappa=\tau=0$ и $\varphi(s) \equiv \varphi_{0}$. В этом случае

$$
\begin{array}{ccc}
a_{1}(s) \in \mathcal{C}^{1}(\mathbb{R}), & a_{2}(s) \in \mathcal{C}^{1}(\mathbb{R}), & b(s) \in \mathcal{C}^{1}(\mathbb{R}), \\
k_{1}(s)=0, & k_{2}(s)=0, & k_{3}(s)=0, \\
F_{1}(s)=0, & F_{2}(s)=0, \quad F_{3}(s)=F_{3}(0) .
\end{array}
$$

Напомним, что $a_{1}(s)>0, a_{2}(s)>0$ и $b(s)>0$ для любого $s$ при фиксированных $a_{1}(0), a_{2}(0)$ и $b(0)$.

С учетом (10) можно явно записать плотность энергии для этого случая:

$$
\mathcal{E}=F_{3}(0)
$$

Из (41) видно, что плотность энергии не зависит от длины дуги и от изгибной и торсионной жесткостей $a_{1}(s), a_{2}(s)$ и $b(s)$. Тогда можно показать, что единственный вклад в энергию упругости дают объемные внешние силы.

4.8. СлУЧАЙ 3б: $\kappa=\tau=0$ и $\varphi(s)$ не является постоянной функцией. В этом случае

$$
\begin{array}{rlrl}
a_{1}(s) \in \mathcal{C}^{1}(\mathbb{R}), & & a_{2}(s) \in \mathcal{C}^{1}(\mathbb{R}), & b(s)=\frac{\beta}{\varphi^{\prime}(s)}, \\
k_{1}(s)=0, & k_{2}(s)=0, & k_{3}(s)=\varphi^{\prime}(s), \\
F_{1}(s)=0, & F_{2}(s)=0, & F_{3}(s)=F_{3}(0) .
\end{array}
$$

Напомним, что $a_{1}(s)>0$ и $a_{2}(s)>0 \forall s$ при фиксированных $a_{1}(0)$ и $a_{2}(0)$; кроме того, $\beta=b(0) \varphi^{\prime}(0)$ должно быть выбрано так, чтобы $b(s)>0$ для любого $s$.

Из (10) вытекает, что плотность энергии для этого случая имеет вид

$$
\mathcal{E}=F_{3}(0)+\frac{\beta}{2} \varphi^{\prime}(s)
$$

Отметим, что плотность энергии (43) зависит от поведения регистра и не зависит от изгибных жесткостей $a_{1}(s)$ и $a_{2}(s)$.

\section{5. ОБРАЩЕНИЕ В НУЛЬ ВНУТРЕННЕГО ТВИСТА}

В данном разделе рассматривается поведение тонких упругих стержней, когда регистр $\varphi(s)$ является постоянным, а именно при обращении в нуль внутреннего твиста $d \varphi(s) / d s=0$. Мы вычислим энергию упругости на один виток и, имея в виду возможные применения к исследованиям биологических и синтетических полимеров, рассмотрим ее как функцию шага спирали при фиксированном радиусе. Тогда с учетом результатов раздела 4 легко видеть, что система (9) допускает решения при $d \varphi(s) / d s=0$ только в перечисленных ниже случаях. Стоит заметить, что многие из представленных здесь результатов являются новыми, а их получение оказалось возможным лишь при использовании аналитических результатов, полученных в работе [6]. 
5.1. СлучАй $\varphi(s) \equiv n \pi / 2, n \in \mathbb{Z}, \tau \neq 0$ и $\kappa \neq 0$. Этот случай, соответствующий случаю 1a, рассмотренному в разделе 4 , описывает два различных вида спиралей Френе с четным или нечетным $n$. Случай $\varphi=\pi$ тривиально переходит в случай $\varphi=0$ путем поворота поперечного сечения на $\pi$. Таким же образом $\varphi=3 \pi / 2$ переходит в случай $\varphi=\pi / 2$. Этот результат уже был получен в работе [12] для постоянных изгибной и торсионной жесткостей.

В $(17)((19))$ жесткость $a_{2}(s)\left(a_{1}(s)\right)$ является постоянной и явно зависит от $\kappa$ и $\tau$, тогда как $F_{2}(0)\left(F_{1}(0)\right)$ играет роль произвольной постоянной.

Наоборот, для исследования зависимости энергии упругости стержня от шага $p$ требуется, чтобы жесткости были независимы от шага. Тогда в (17) ((19)) полагаем

$$
\begin{gathered}
F_{2}(0)=(-1)^{m}\left(b_{0}-a_{2}\right) \frac{r c}{\left(r^{2}+c^{2}\right)^{2}} \\
\left(F_{1}(0)=(-1)^{m+1}\left(b_{0}-a_{1}\right) \frac{r c}{\left(r^{2}+c^{2}\right)^{2}}\right),
\end{gathered}
$$

где $m$ и $b_{0}$ приведены в п. 4.1 .1 (п. 4.1.2), причем $F_{2}(0)\left(F_{1}(0)\right)$ зависят от кривизны и кручения так, чтобы изгибная жесткость $a_{2}(s)\left(a_{1}(s)\right)$ оставалась постоянной при всех значениях шага, $a_{2}(s) \equiv a_{2}\left(a_{1}(s) \equiv a_{1}\right)$. Тогда энергия на один виток для спирали первого типа (т.е. при четном $n$ ) есть

$$
E=\pi \frac{a_{2} r^{2}+\left(3 b_{0}-2 a_{2}\right) c^{2}}{\left(c^{2}+r^{2}\right)^{3 / 2}}
$$

а энергия на один виток для спирали второго типа (т.е. при нечетном $n$ ) есть

$$
E=\pi \frac{a_{1} r^{2}+\left(3 b_{0}-2 a_{1}\right) c^{2}}{\left(c^{2}+r^{2}\right)^{3 / 2}} .
$$

При фиксированных значениях радиуса $r$ энергия на один виток спирали (45) $((46))$ стремится к нулю при $p \rightarrow \infty$, но принимает положительное значение при $p \rightarrow 0$ :

$$
E=\frac{\pi a_{2}}{r} \quad\left(E=\frac{\pi a_{1}}{r}\right)
$$

в соответствии с интуитивным представлением о том, что незакрученная конфигурация спирали более устойчива, чем закрученная конфигурация свернувшейся спирали. Важно заметить, что этот анализ применим для идеализированной спирали, представленной своей центральной линией, без учета эффектов, вызванных существованием конечного (и ненулевого) поперечного сечения, что исключает полное вырождение центральной линии в окружность, (подробнее см. [13]). Более удивителен тот факт, что энергия упругости (45) ((46)), рассматриваемая как функция шага, может иметь единственный глобальный минимум $E_{\mathrm{m}}<0$ при фиксированном значении $r$ :

$$
E_{\mathrm{m}}=-\frac{2 \pi\left(2 a_{2}-3 b_{0}\right)^{3 / 2}}{9 r \sqrt{a_{2}-b_{0}}} \quad\left(E_{\mathrm{m}}=-\frac{2 \pi\left(2 a_{1}-3 b_{0}\right)^{3 / 2}}{9 r \sqrt{a_{1}-b_{0}}}\right)
$$


при

$$
p_{\mathrm{m}}=r \sqrt{\frac{7 a_{2}-6 b_{0}}{2 a_{2}-3 b_{0}}} \quad\left(p_{\mathrm{m}}=r \sqrt{\frac{7 a_{1}-6 b_{0}}{2 a_{1}-3 b_{0}}}\right)
$$

и при подходящем выборе параметров $b_{0}$ и $a_{2}\left(a_{1}\right)$

$$
b_{0}<\frac{2}{3} a_{2} \quad\left(b_{0}<\frac{2}{3} a_{1}\right) .
$$

Это приводит к неожиданной физической интерпретации существования устойчивой спиральной конфигурации с конечными радиусом и шагом.

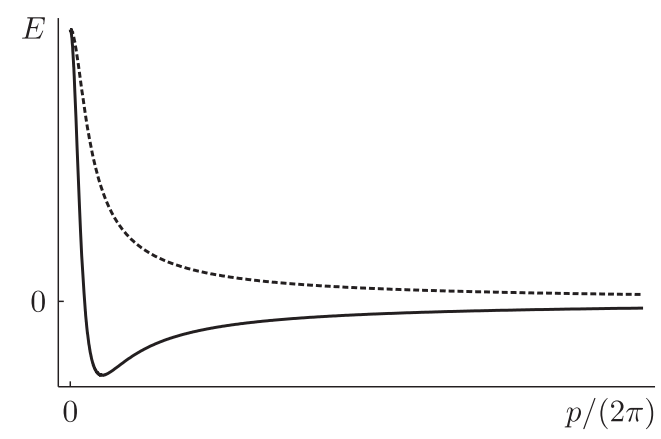

Рис. 3. Энергетические диаграммы при $r \neq 0, b_{0} \geqslant 2 a_{2} / 3$ (пунктир) и $b_{0}<2 a_{2} / 3$ (сплошная линия).

5.2. $\varphi(s) \equiv \varphi_{0}, \varphi_{0} \neq n \pi / 2, n \in \mathbb{Z}, \kappa \neq 0$ и $\tau \neq 0$. Этот случай соответствует случаю 1в, рассмотренному в разделе 4 , когда $\varphi$ - вещественная постоянная.

В выражении (25) жесткости $a_{1}(s), a_{2}(s)$ и $b(s)$ являются постоянными и явно зависят от $\kappa$ и $\tau$. Аналогично п. 5.1 для исследования энергии упругости стержня в зависимости от шага $p$ требуется, чтобы жесткости были независимы от шага. Тогда в (25) можно положить

$$
\beta_{0}=a_{0} \tau \quad \beta_{1}=b_{0} \tau,
$$

где $a_{0}$ и $b_{0}$ - произвольные вещественные постоянные такие, что выполнено (27), а $a_{1}(s), a_{2}(s)$ и $b(s)$ остаются постоянными при всех значениях шага. Тогда, используя (28) и (14), получаем, что энергия на один виток спирали есть

$$
E=\pi \frac{a_{0} r^{2}+\left(3 b_{0}-2 a_{0}\right) c^{2}}{\left(c^{2}+r^{2}\right)^{3 / 2}} .
$$

Очевидно, что (50) переходит в (45) ((46)) после замены $a_{0} \rightarrow a_{2}\left(a_{0} \rightarrow a_{1}\right)$. Тогда подробное рассмотрение выражения (50) можно выполнить, следуя анализу, проведенному в п. 5.1. Тем не менее существует фундаментальное различие между рассмотренным выше случаем и предыдущими: если $\varphi=m \pi(\varphi=m \pi+\pi / 2)$, $m \in \mathbb{Z}$, то торсионная жесткость $b(s)$ и изгибная жесткость $a_{2}(s)\left(a_{1}(s)\right)$ постоянны и $a_{1}(s) \in \mathcal{C}^{1}(\mathbb{R})\left(a_{2}(s) \in \mathcal{C}^{1}(\mathbb{R})\right)$, но если $\varphi \neq n \pi / 2, n \in \mathbb{Z}$, то эти жесткости определяются выражением (25). 
5.3. $\varphi(s) \equiv n \pi / 2, n \in \mathbb{Z}, \tau=0$ и $\kappa \neq 0$. Этот случай, соответствующий случаю $2 \mathrm{a}$, рассмотренному в разделе 4 , описывает кольцеподобный стержень. Случай $\varphi=\pi$ тривиально переходит в случай $\varphi=0$ путем поворота поперечного сечения на $\pi$; таким же образом $\varphi=3 \pi / 2$ переходит в случай $\varphi=\pi / 2$. Этот результат уже был получен в работе [12] для постоянных изгибной и торсионной жесткостей.

Интегрируя плотность энергии (30) (или, эквивалентно, (32)) по витку, получаем энергию упругости на один виток в виде

$$
E=\frac{\pi a_{0}}{r}
$$

где выражение для $a_{0}$ приведено в п. 4.1.1. Стоит отметить, что (51) совпадает с выражением для энергии упругости (45) ((46)) в пределе $c \rightarrow 0$, если $b_{0}$ из (45) ((46)) полагается равным $a_{0}$ из $(29)((31))$.

5.4. $\varphi(s) \equiv \varphi_{0}, \varphi_{0} \neq n \pi / 2, n \in \mathbb{Z}, \kappa \neq 0$ и $\tau=0$. Этот случай, соответствующий случаю 2б, рассмотренному в разделе 4 , описывает кольцеподобный стержень с углом общего положения $\varphi \neq n \pi / 2$. Конфигурацию этого типа можно расссматривать как вырождение круговой спирали.

Интегрируя плотность энергии (35) по витку, получаем энергию упругости на один виток

$$
E=\frac{\pi \alpha_{0}}{r} .
$$

Стоит заметить, что выражение (52) совпадает с выражением для энергии упругости (51) в пределе $c \rightarrow 0$, если $b_{0}$ из (52) полагается равным $\alpha_{0}$ из (33).

5.5. $\varphi(s) \equiv \varphi_{0}$ и $\kappa=\tau=0$. Этот случай отвечает случаю За, рассмотренному в разделе 4 , и описывает прямой стержень, соответствующий углу общего положения $\varphi(s) \equiv \varphi_{0}$. Конфигурацию этого типа также можно рассматривать как вырождение круговой спирали. Этот случай рассматривается в работе [15].

Для стержня длины $2 \ell$ можно получить энергию упругости из (41) с помощью формулы (15): единственный вклад возникает из-за касательной компоненты объемной внешней силы вдоль центральной линии.

\section{6. ЗАКЛЮЧЕНИЕ}

В настоящей работе рассмотрена плотность энергии упругости для круговых спиральных решений статических уравнений Кирхгофа для тонкого упругого стержня. Мы подошли к изучению статической системы Кирхгофа как к обратной задаче, предполагая, что центральная линия стержня имеет конфигурацию круговой спирали с фиксированным поведением регистра; в этом случае мы нашли соответствующие жесткости и объемные внешние силы. Используя результаты, полученные и описанные в работе [6], для каждого из восьми случаев, составляющих общее решение этой обратной задачи, мы вычислили плотность энергии упругости. Далее мы применили результаты к характеризации тонких упругих стержней с обращающимся в нуль внутренним твистом. Оказалось, что когда кривизна и кручение конечны и не обращаются в нуль, а регистр не зависит от длины дуги, энергия на 
один виток имеет минимум при стремлении шага к бесконечности и не расходится при стремлении шага к нулю. Более того, при подходящем выборе жесткостей энергия упругости может достигать глобального минимума при конечном значении шага. Эти результаты дают возможность описать механизм отбора, выделяющего устойчивую конфигурацию тонкого упругого стержня, центральная линия которого описывает круговую спираль, при условии, что его физические параметры фиксированы.

\section{Список литературы}

[1] R. Improta, V. Barone, K. N. Kudin, G.E. Scuseria, J. Chem. Phys., 114:6 (2001), 2541-2549.

[2] J. S̆poner, F. Lankaš (eds.), Computational Studies of RNA and DNA, Challenges and Advances in Computational Chemistry and Physics, 2, Springer, New York, 2006.

[3] R. Improta, V. Barone, K. N. Kudin, G. E. Scuseria, J. Am. Chem. Soc., 123:14 (2001), 3311-3322.

[4] R. Improta, F. Mele, O. Crescenzi, C. Benzi, V. Barone, J. Am. Chem. Soc., 124:26 (2002), $7857-7865$.

[5] H. Gohlke, M. F. Thorpe, Biophysical J., 91:6 (2006), 2115-2120.

[6] M. Argeri, V. Barone, S. De Lillo, G. Lupo, M. Sommacal, Physica D, 238 (2009), 1031-1049.

[7] E. Langella, R. Improta, V. Barone, Biophysical J., 87:6 (2004), 3623-3632.

[8] A. E. H. Love, A Treatise on the Mathematical Theory of Elasticity, Dover, New York, 1944.

[9] S. S. Antman, Nonlinear Problems of Elasticity, Appl. Math. Sci., 107, Springer, New York, 1995.

[10] Г. Кирхгоф, Механика, Лекции по математической физике, АН СССР, М., 1962.

[11] S. R. Sanghani, K. Zakrzewska, S. C. Harvey, R. Lavery, Nucleic Acids Res., 24:9 (1996), 1632-1637.

[12] A. Goriely, M. Nizette, M. Tabor, J. Nonlinear Sci., 11:1 (2001), 3-45.

[13] N. Chouaieb, A. Goriely, J.H. Maddocks, Proc. Natl. Acad. Sci. USA, 103:25 (2006), 9398-9403.

[14] A.F. Bower, Applied mechanics of solids, http://solidmechanics.org, 2008.

[15] S. Zhang, X. Zuo, M. Xia, S. Zhao, E. Zhang, Phys. Rev. E, 70:5 (2004), 051902. 